\title{
Modelling the Effects of Paclitaxel and Cisplatin on Breast and Ovarian Cancer
}

\author{
JOHN CARL PANETTA ${ }^{a, *}$, MARK A. J. CHAPLAIN ${ }^{b}$ and DAVID CAMERON ${ }^{c}$
}

${ }^{a}$ Pharmaceutical Sciences Department, St. Jude Children's Research Hospital, 332 N. Lauderdale St., Memphis TN, 38105-2794; ${ }^{\mathrm{b}}$ Department of Mathematics, University of Dundee, Dundee DDI $4 \mathrm{HN}$; ' Department of Oncology, University of Edinburgh, Western General Hospital, Edinburgh EH4 $2 X U$

(Received 11 June 1999; In final form 31 January 2000)

\begin{abstract}
The two drugs, Paclitaxel and Cisplatin, have important roles in the treatment of breast and ovarian cancer, with the combination currently considered the optimum first line chemotherapy of epithelial ovarian cancer. There has been a variety of experimental and clinical studies to try to determine the most effective method to deliver these drugs. These studies consistently show that giving Paclitaxel prior to Cisplatin is the more effective regimen. However, the reasons why are not fully understood. Therefore, we have developed a mathematical model to describe and predict the effects of these two drugs. This model takes into account the cytotoxic effects of the drugs on the cell-cycle and the pharmacodynamic and pharmacokinetic effects of the drugs on each other. The model agrees with the experimental and clinical studies which show that Paclitaxel given prior to Cisplatin is the better combination and, in addition, the model also predicts more effective treatment regimens. These include conditions on the time between doses and the dosing of each of the drugs.
\end{abstract}

Keywords: cancer, combination chemotherapy, Paclitaxel, Cisplatin, ordinary differential equations

\section{INTRODUCTION}

Paclitaxel (Taxol - Bristol-Myers Squibb Company) and Cisplatin are both cell-cycle specific chemotherapeutic drugs. Each is currently being used to treat both breast and ovarian cancer along with other types of cancer. Currently Taxol is being used alone as a monotherapy and also in combination with Cisplatin. Overall, these treatments are having a $20 \%-61.5 \%$ complete or partial response in breast cancer and a $16 \%-48 \%$ complete or partial response in ovarian cancer (Spencer and Faulds [19]).
However, two important questions remain incompletely answered:

- What is the optimal method of scheduling this combination of drugs?

- What causes one schedule of treatment to be more effective than another?

Taxol is an antimitotic agent which promotes the assembly of intracellular tubulin. However, unlike other antimitotic agents it then stabilizes the resulting microtubules which prevents the cells from dividing correctly (Arbuck et al. [1]; Spencer and Faulds [19]). In contrast to this, Cisplatin is a platinum coordination complex which disrupts DNA

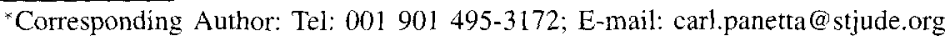


synthesis. It has been shown that these two drugs are non-cross-resistant (Engblom et al. [7]; Spencer and Faulds [19]) at least in part because they each act on a different stage of the cell-cycle.

There is a variety of in vitro and in vivo studies that have attempted to determine acceptable sequencing of these two drugs to provide at least additive results $[7,9,11,12,14,16,20]$. The overall consensus of these studies (with the exception of [11] which was inconclusive) is that the sequencing of these two drugs is very important and in general they all conclude that when Taxol is administered prior to Cisplatin there are additive or superadditive effects (sometimes called synergistic), but when they are administered together or in reverse order there are antagonistic results (less than additive). However, when Taxol is administered prior to Cisplatin, it is still not clear, what the interval of time should be between the administration of each drug, and, even more importantly, what the cause of this sequence dependence is. Some suggest that the differences in sequencing are due to the fact that the two drugs are active in different phases of the cell-cycle (Liebmann et al. [12]). That is, Cisplatin blocks cells from entering the Mphase and is therefore antagonistic to the M-phase drug Taxol. But Jekunen et al. [9] suggest that the differences in sequencing are not due to the cell kinetics, but are more likely to be of a molecular nature, while Milross et al. [14] suggest that those cells surviving a dose of Cisplatin could then be more sensitive to Taxol. Also DeVos et al. [5] showed a significant reduction in the intracellular Cisplatin concentration in leukocytes in the presence of Tween 80 and Cremophor EL (which is co-administered with Taxol), although this observation was only made in hematopoietic cells.

In this paper we will examine and model three experimentally observed interactions between Cisplatin and Taxol (the mathematical form of these interactions is discussed in Section 2 and summarized in Table I). These are as follows: (i) Vanhoefer et al. [20] observed that when Cisplatin preceded Taxol there was a decreased intracellular retention of Taxol. Furthermore they go on to report that the cell-cycle changes caused by Cisplatin and Taxol are not sufficient to explain the antagonistic results observed with concurrent shortterm exposure to both drugs. (ii) Another interesting pharmacodynamic interaction between Taxol and Cisplatin was observed by Jones et al. [10]. They reported that Taxol increases the apoptotic rate. (iii) Finally, Rowinsky et al. [17] concluded from pharmacokinetic studies that the difference in drug sequencing could be due to a $25 \%$ lower Taxol clearance rate when Cisplatin precedes Taxol. Their data suggest that the sequence dependence is more likely to be due to pharmacokinetics and pharmacodynamics rather than cell cycle effects.

In the following sections of this paper we develop a mathematical model that describes the effects of the two drugs (Taxol, Cisplatin) on the growth of cancer cells in vitro - i.e. for cells undergoing exponential growth. This model will distinguish

TABLE I Functional forms of the model parameters. The parameters are scaled values (thus non-dimensional) unless indicated

\begin{tabular}{|c|c|c|c|}
\hline Parameter & Value or Functional form & Reference & Sections Discussed \\
\hline$\delta_{t}(C, T)$ & $\frac{\delta_{t}}{1+\theta_{t} C}, \delta_{t}=1.0, \theta_{t}=0-50($ see Figures 7,9$)$ & {$[20]$} & $3.3 .3-3.3 .4$ \\
\hline$\delta_{c}(C, T)$ & $\delta_{c}=1.0($ fixed $)$ & & \\
\hline $\begin{array}{l}\gamma_{m}(T) \\
\gamma_{s}(T)\end{array}$ & $\begin{array}{c}\gamma_{m}\left[1+\frac{\gamma_{m 2} \theta_{m} T}{1+\theta_{m} T}\right], \gamma_{m}=0.5, \gamma_{m 2}=4.0, \theta_{m}=0-50(\text { see Figure } 8) \\
\gamma_{s}=0.5(\text { fixed })\end{array}$ & {$[10]$} & $3.3 .3-3.3 .4$ \\
\hline $\begin{array}{l}\gamma_{s}(1) \\
\eta\end{array}$ & $\begin{aligned} \gamma_{s} & =0.5 \text { (fixed) } \\
\eta & =0.1(\text { fixed except in Figure 10) }\end{aligned}$ & & \\
\hline$\lambda_{t}(C, T)$ & $\frac{\lambda_{t}}{1+\theta_{\lambda} \bar{C}}, \lambda_{t}=2.0, \theta_{\lambda}=0-50$ (see Figure 11) & {$[17]$} & 3.3 .4 \\
\hline $\begin{array}{l}\lambda_{c}(C, T) \\
D \\
a\end{array}$ & $\begin{array}{l}\lambda_{c}=2.0 \text { (fixed) (May be an increasing function of Taxol.) } \\
\text { Taxol: } D=0-60 \text {, Cisplatin: } D=0-100 \text { (see Figures } 5,10 \text { ) } \\
\text { Taxol: } a=1.0 \text { (days), Cisplatin: } a=0.08 \overline{3} \text { (days) or }(2 \mathrm{hrs} \text { ) }\end{array}$ & [5] & $3.3 .1-3.3 .3$ \\
\hline
\end{tabular}


between cells in the $G_{1} / S$-phase, $G_{2} / M$-phase, and also permits a subset of cells to be drug sensitized. In addition to qualitatively matching the results from various clinical studies, we use the model to try to help answer several important questions from a chemotherapeutic viewpoint:

1. Does the sequence interaction of the two drugs depend on the cell-cycle and/or pharmacokinetics/pharmacodynamics? That is, are the changes in the cell-cycle due to the drugs sufficient to explain the synergistic and antagonistic results observed?

2. What sequencing does the model suggest gives the best cytotoxicity?

3. How do the model results compare to current clinical practices in delivering this combination of drugs?

\section{THE MATHEMATICAL MODEL}

The main dynamics that a mathematical model describing the growth and control of cancer treated with Taxol and Cisplatin should contain are those describing the rates of movement of the cancer cells through the cell-cycle, in particular the active phases $G_{1}, S, G_{2}$, and $M$. In designing this model we will make several justifiable assumptions:

1. We will only consider linear growth kinetics (i.e. the growth parameters are constant). Since we are modelling in vitro growth kinetics then a legitimate assumption is that the cells will grow exponentially. This is consistent with the in vitro studies referenced to above which were performed with cells growing exponentially.

2. We will combine the cell-cycle phases $G_{1}$ and $S$ and also $G_{2}$ and $M$ to form two separate compartments $-G_{1} / S$ and $G_{2} / M$. The primary reason the cell-cycle is grouped in this manner is theoretical - the Taxol only affects cells in mitosis and the Cisplatin affects DNA replication. Secondly, $G_{2} / M$ cannot be distinguished in FACS analysis (and therefore cannot be distinguished in the subsequent confirmatory cell-line studies). Finally, this will reduce the number of equations that we consider.

3. We assume that Cisplatin damages the DNA in all phases of the cell-cycle and damaged cells cannot proceed past the $S$-phase without repairing their DNA.

4. Taxol only damages cells in the $M$-phase.

5. Damaged cells die due to apoptosis, at a constant rate.

6. The drug effects will be functions of Cisplatin and Taxol concentration to account for the various observed pharmacokinetic/pharmacodynamic effects these two drugs have on each other.

Therefore, we develop a model taking into account the above assumptions which define four distinct compartments in the cell cycle: $G_{1} / S$, in which cells are resistant to Taxol; $G_{2} / M$, in which cells are sensitive to Taxol; sensitized $G_{1} / S$, for Cisplatin damaged cells; and sensitized $G_{2} / M$, for Taxol damaged cells.

The basic form of the model is described diagrammatically in Figure 1.

To translate this schematic figure into a set of ordinary differential equations we use a "law of mass action" approach and examine the rates of change of each of the cell types. The variables used

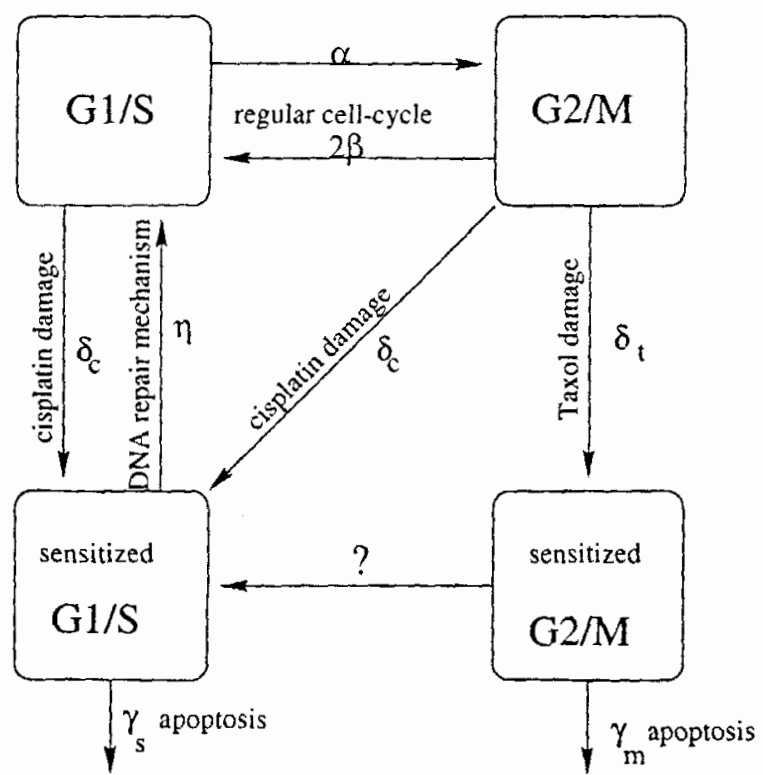

FIGURE 1 The Cell-Cycle With Drug Treatment. 
in the model are defined as follows: $S$, the number of cells in the $G_{1}$ - and $S$-phases; $M$, the number of cells in the $G_{2}$ - and $M$-phases; $S_{s}$, the number of Cisplatin-damaged cells; $M_{s}$, the number of Taxoldamaged cells; $T$, Taxol concentration; and, $C$, Cisplatin concentration. For example if we examine the rate of change of the number of cells in the $G 1 / S$ compartment, $d S / d t$, we see from the diagram that there is a loss of cells at rate $\alpha$ into the $G 2 / M$ compartment; a loss of cells at rate $\delta_{c}$ into the $G 1 / S$ compartment; a gain of cells at a rate $2 \beta$ from the $G 2 / M$ compartment and a gain of cells at a rate $\eta$ from the $G 1 / S$ compartment. Combining these facts leads to Equation (1) below. In a similar manner, we can derive the equations describing the other three cell types in the model. Therefore, mathematically the above figure translates to the following system of ordinary differential Equations (1)-(4):

$$
\begin{aligned}
\frac{\mathrm{d} S}{\mathrm{~d} t}= & -\left[\alpha+\delta_{r}(C, T) C\right] S+2 \beta M+\eta S_{s}, \\
\frac{\mathrm{d} M}{\mathrm{~d} t}= & \alpha S-\left[\beta+\delta_{t}(C, T) T+\delta_{c}(C, T), C\right] M, \\
\frac{\mathrm{d} S_{s}}{\mathrm{~d} t}= & {\left[\delta_{c}(C, T) C\right] S+\left[\delta_{c}(C, T) C\right] M } \\
& -\left(\eta+\gamma_{s}(T)\right) S_{s}, \\
\frac{\mathrm{d} M_{s}}{\mathrm{~d} t}= & {\left[\delta_{t}(C, T) T\right] M-\gamma_{m}(T) M_{s} . }
\end{aligned}
$$

Next, the pharmacokinetics of the model governing the rates of change of the Taxol and Cisplatin concentrations are governed by Equations (5) and $(6)$ :

$$
\begin{aligned}
& \frac{\mathrm{d} T}{\mathrm{~d} t}=s_{t}(t)-\lambda_{t}(C, T) T, \\
& \frac{\mathrm{d} C}{\mathrm{~d} t}=s_{C}(t)-\lambda_{c}(C, T) C .
\end{aligned}
$$

The parameters of Equations (1)-(6) are defined as follows. $\alpha$ is the rate of transition from $S$ to $M . \beta$ is the rate of transition from $M$ to $S$ where the factor $2 \beta$ in Equation (2) accounts for the cell division. $\delta_{i}(C, T), i=c, t$, represents the effectiveness for each of the drugs in "killing" the cancer cells. It may be a function of the drug concentrations to account for possible pharmacodynamic interactions.
For example, to account for the decreased uptake of Taxol in the presence of Cisplatin (as observed in Vanhoefer et al. [20]) we will consider $\delta_{t}(C, T)$ to be of the form

$$
\delta_{l}(C, T)=\frac{\delta_{t}}{1+\theta_{t} C}
$$

The apoptosis rates from each of the drug sensitized compartments is represented by $\gamma_{i}(T), i=$ $s, m$. These can be increasing functions of Taxol dose (as observed in Jones et al. [10]) and we will consider the form

$$
\gamma_{i}(T)=\gamma_{i}\left[1+\frac{\gamma_{i 2} \theta_{i} T}{1+\theta_{i} T}\right], \quad i=s, m .
$$

The rate of DNA repair is represented by $\eta$. $s_{i}(t), i=t, c$, are the drug source terms. They are of the form of the step function

$$
s_{i}(t)= \begin{cases}D, & 0 \leq t \leq a \\ 0, & a<t \leq e n d\end{cases}
$$

where $D$ is the drug concentration, $a$ is the drug infusion time, and end is the end time of the experiment. Finally, $\lambda_{i}(C, T), i=t, c$, represent the rate of decay of the drug from the system. These are functions of the drug concentrations to account for possible pharmacokinetic interactions, such as the decreased clearance of Taxol in the presence of Cisplatin observed by Rowinsky et al. [17]. Thus, we will consider its form to be

$$
\lambda_{t}(C, T)=\frac{\lambda_{1}}{1+\theta_{\lambda} C}
$$

Table I summarizes all the above parameter information including values or ranges of values for the parameters and which section they are discussed.

\section{NUMERICAL SIMULATIONS}

\subsection{Growth Model Parameter Estimation}

We estimate the growth parameters $\alpha$ and $\beta$ using the methods discussed in Panetta [15]. Here we take the doubling time to be between 0.25 and 6 days and the percentage of cells in $G_{1} / S$-phase 
TABLE II Model Parameters

\begin{tabular}{lll}
\hline $\begin{array}{l}\text { Doubling Time }=2.3 \text { days, Units }=\text { days } \\
\text { MCF- } 7 / \text { LY2 }\end{array}$ & $\alpha=0.762$ & $\beta=0.625$ \\
Cell-Cycle Distribution & $G_{1} / S$-phase $=55 \%$ & $G_{2} / M$-phase $=45 \%$ \\
Doubling Time $=1.2$ days, Units $=$ days $^{-1}$ & & \\
A2780 & $\alpha=0.9755$ & $\beta=2.132$ \\
Cell-Cycle Distribution & $G_{1} / S$-phase $=72 \%$ & $G_{2} / M$-phase $=28 \%$
\end{tabular}
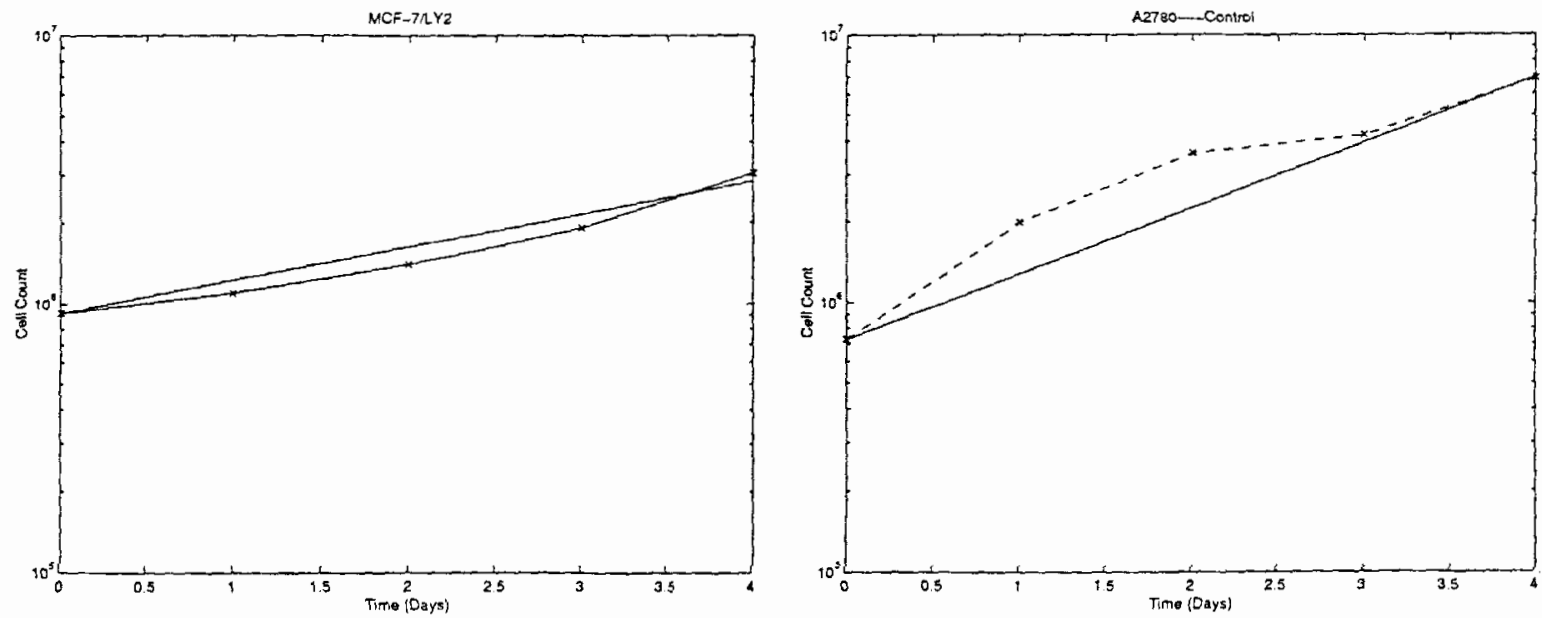

FIGURE 2 MCF-7/LY2 (Left) and A2780 (Right) growth data. " $x$ _ _ represent the experimental data and " _. " represent model results.

to be between $50-80 \%$ and $G_{2} / M$ to be between $20-50 \%$. Using data on the breast cancer cell line MCF-7/LY2 and ovarian cancer cell line A2780 (obtained from Billy Day, Ph.D. - University of Pittsburgh) we obtain appropriate model parameters (see Table II). Figure 2 compares the MCF-7/LY2 and A2780 data to the estimated parameters. As can be seen, the fit is acceptable. Using the parameters in Tables I and II, the system of ordinary differential Equations (1)-(6) was solved numerically using standard Runge-Kutta methods, the results of which are presented in Section 3.3 .

\subsection{Methods of Measuring the Combination Effects}

The question that we ask for each combination of drugs is: are the effects additive, super-additive (synergistic), or sub-additive (antagonistic)? There are several measures used to determine additivity in combination therapy. Some measures include the isobologram, combination index, or response surface method. (see Chou and Talalay [4], Berenbaum [2], and Greco et al. [8] respectively for more details on these methods.) We determine the combination effect by considering the absolute growth delay (AGD) caused by the dose (see Milross et al. [14]). The AGD is defined as the difference in the time it takes for the treated cell mass to grow to a specified level and the time it takes for the untreated cell mass (control) to grow to the same level. For the AGD to be consistent, this level should be chosen such that the cell populations are in their exponential phase (i.e. as seen in Figure 3, the solutions should be past the transient part of their solutions).

\subsection{Model Results}

Now we will use the model to help indicate effective regimens of delivering the combination of 


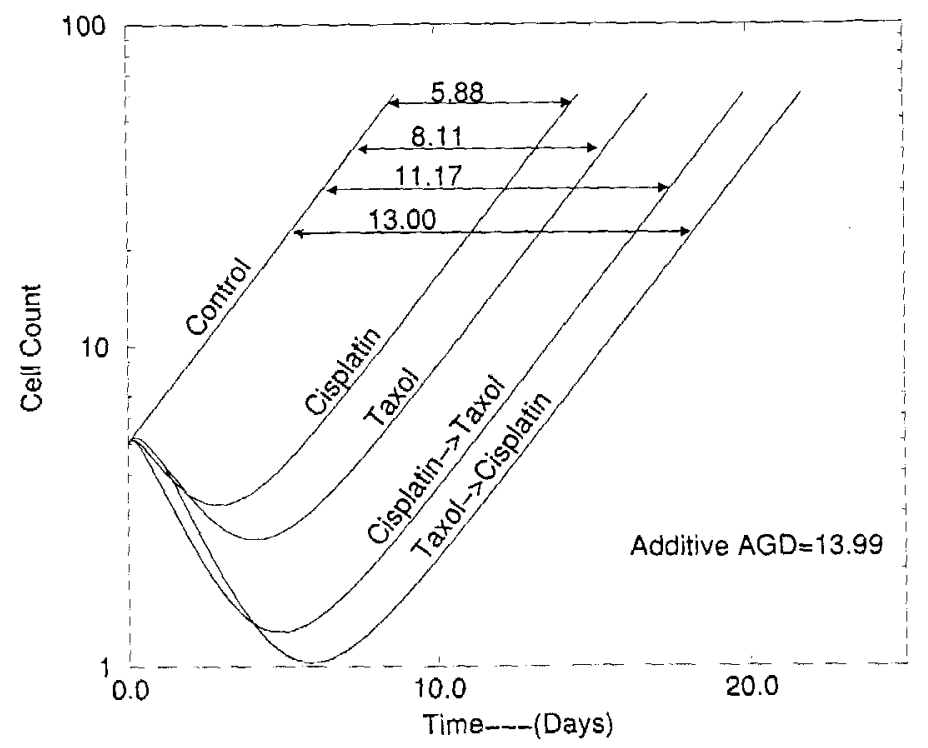

FIGURE 3 MCF-7/LY2 Parameters.

Taxol and Cisplatin. First we consider the model results without pharmacodynamic or pharmacokinetic interactions. Here we would like to know if, considering only the cell-cycle kinetics, the model can be at least qualitatively related to known experimental and clinical information. Then we consider how each of the observed pharmacodynamic or pharmacokinetic interactions (listed in Table I) affect the model results, first separately and then in combination. In all cases we will give Taxol for 24 hours $(a=1$ in Equation 9) and Cisplatin for 2 hours ( $a=0.08 \overline{3}$ in Equation 9) which compares to common infusion times for these drugs.

\subsubsection{No pharmacodynamics/pharmacokinetics}

First we consider the case where the only kinetic effects are those of the cell cycle. Thus in this section we assume that the drugs do not interact and therefore do not alter the effectiveness of each other. Therefore, the functions $\delta_{l}, \delta_{c}, \gamma_{m}, \gamma_{s}$, $\lambda_{t}, \lambda_{c}$ are all constant (i.e. $\theta_{i}=0, i=t, c, s, m, \lambda$ ). Figure 3 shows the differences when Taxol precedes Cisplatin given a fixed dose and vice versa. Note that for this example Taxol preceding Cisplatin is the better combination since it has a larger AGD. This is qualitatively what has been observed experimentally. The reason for this sequence dependence is the difference in cell-cycle specificity between the two drugs. Thus, if Cisplatin is given first, it blocks cells at S-phase preventing them from entering $\mathrm{M}$-phase which is the effect site of Taxol.

Next we consider how the time between doses affects the additivity of the combinations. Figure 4 indicates again that Taxol given before Cisplatin is the better of the two combinations. Note that since the infusion time of Taxol is fixed at 1 day, then for the Taxol $\rightarrow$ Cisplatin regimen, a time between dose of -1 days is equivalent to the two drugs being administered simultaneously and a time between dose of $-2 / 3$ of a day represents starting Cisplatin $1 / 3$ of a day into the Taxol infusion. Figure 4 also shows that when the drugs are administered simultaneously we observe the greatest antagonism. However, at best the model only indicates an additive response for the combination Taxol $\rightarrow$ Cisplatin, when the drugs are administered at least two days apart.

Finally, we consider how altering the dose level for each drug affects the additivity. Figure 5 shows 

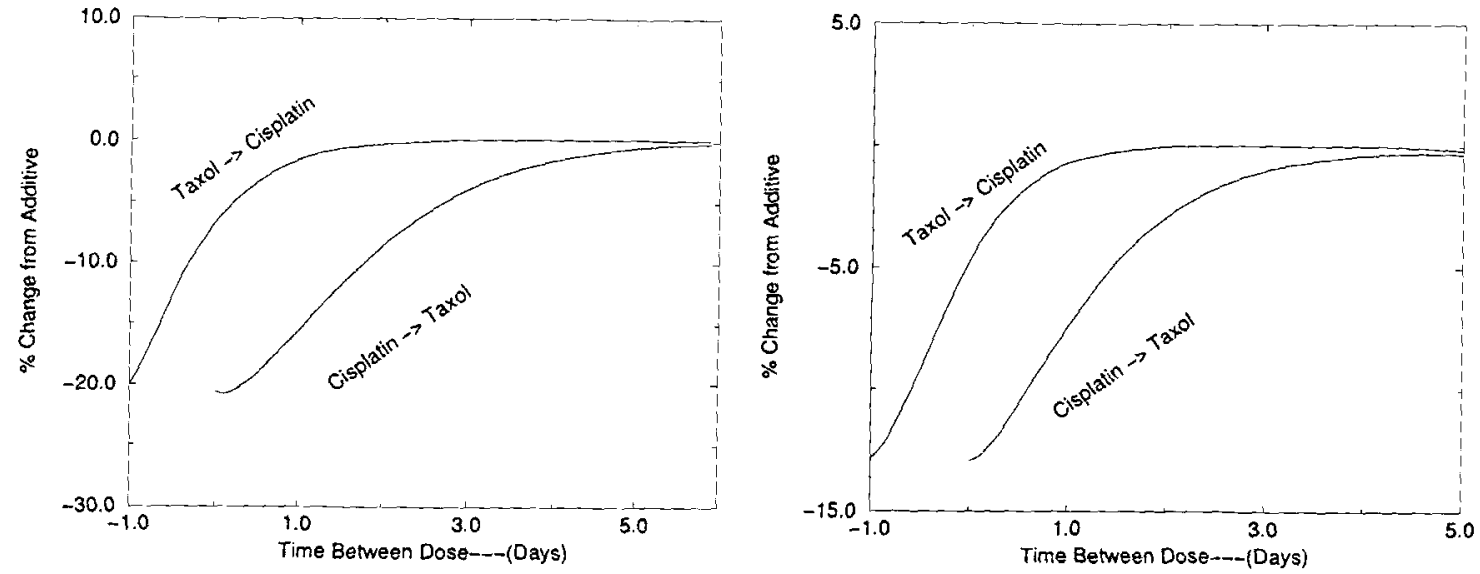

FIGURE 4 Time between dose. MCF-7/LY2 (Left), A2780 (Right). Note: the time between dose of 0 indicates that one dose follows the other immediately and the time between dose of -1 indicates that the two drugs are being given simultaneously since Taxol's infusion time is 1 day.
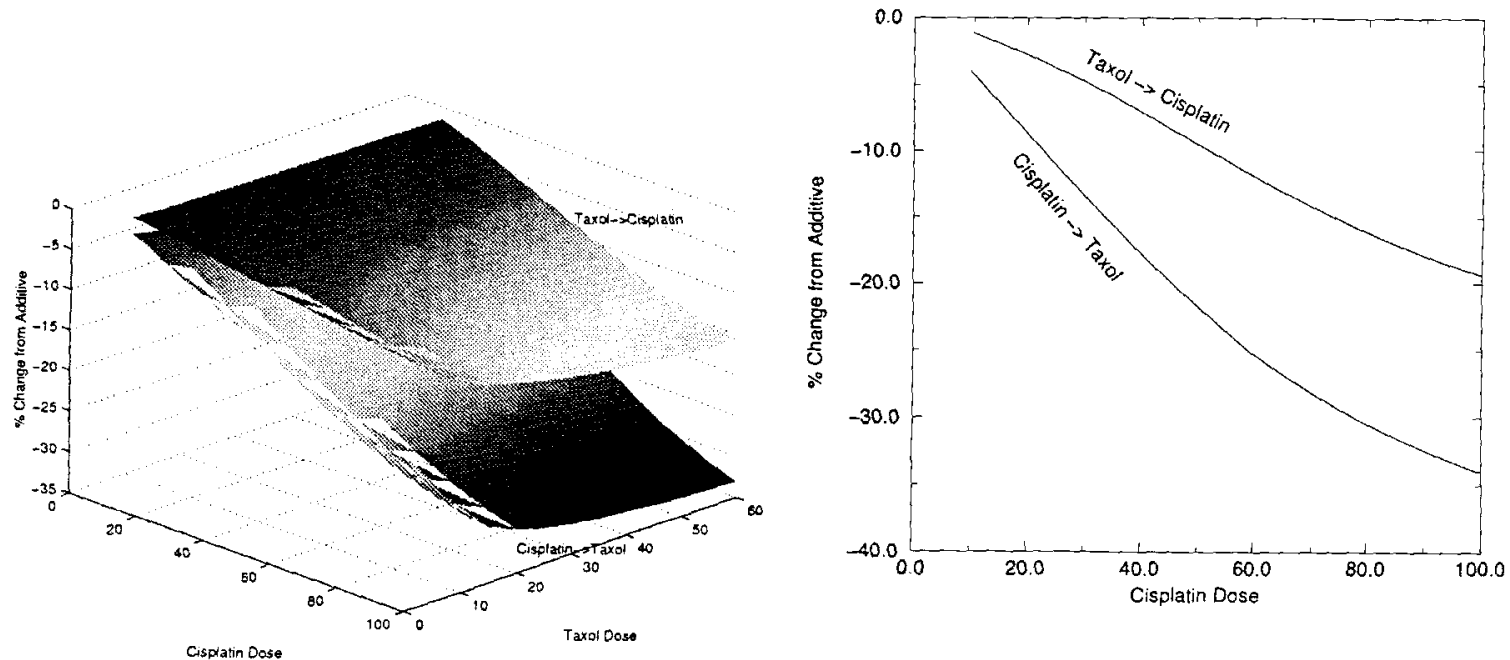

FIGURE 5 MCF-7/LY2 parameters. The ratio between Taxol and Cisplatin dose is kept fixed in the right figure.

that in all cases giving Taxol before Cisplatin is the better combination, but in no case is there synergy and as the doses are increased the level of antagonism is increased.

\subsubsection{Doubling time}

Now we observe the sensitivity of the results to changes in the doubling time of the cells (changes in $\alpha$ and $\beta$ ) while fixing the other parameters. In Figure 6 we observe that for doubling times in the range of 0.25 days to 7 days (a reasonable range for the in vitro studies) Taxol prior to Cisplatin is consistently the better regimen. Also we observe that the doubling time has little effect on the percent change from additivity, indicating that changes in the growth rate have only minor effects on treatment efficacy.

When we do not consider the possibility of pharmacodynamics or pharmacokinetics the model always indicates (as suggested by experimental data) that giving Taxol before Cisplatin is the better combination. And, giving some time between treatments improves additivity. But the model fails to show any synergy - at best it shows additivity when Taxol precedes Cisplatin. This indicates the possible 

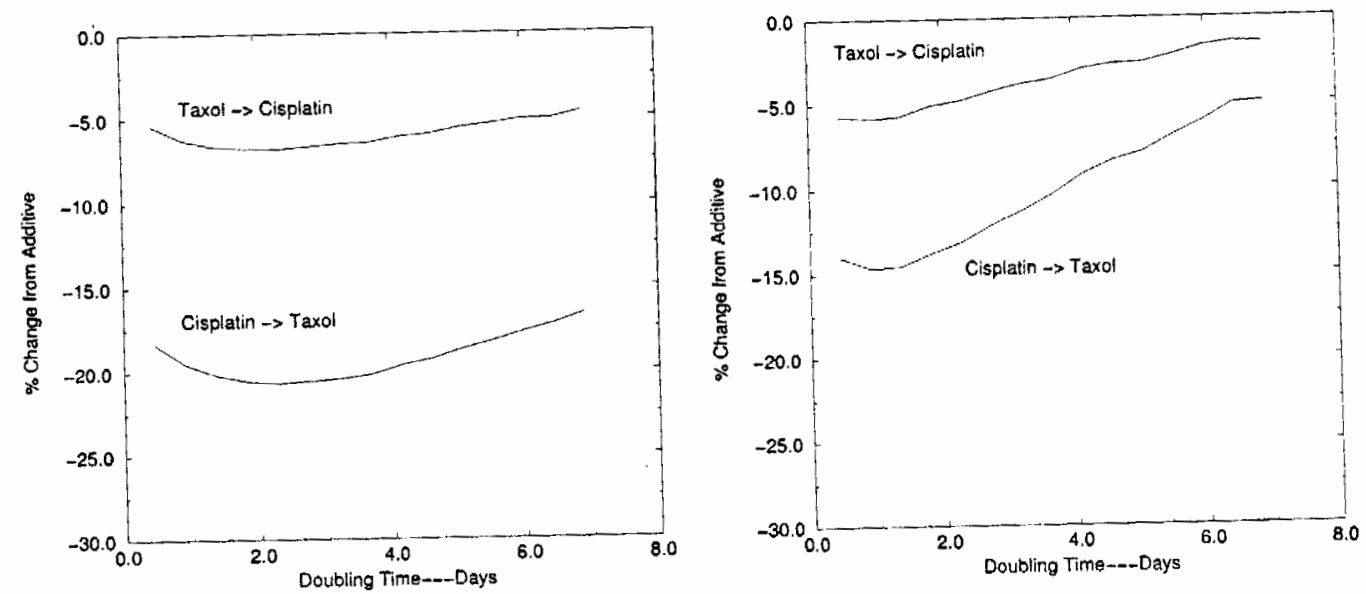

FIGURE 6 The effects of altering the doubling time on the MCF-7/LY2 (Left) and A2780 (Right) cell-line.

importance of the pharmacodynamics and/or pharmacokinetic interactions in producing true synergy.

\subsubsection{Pharmacodynamics}

In this section we include some of the known pharmacodynamic effects into the model. First, we include the effect described by Vanhoefer et al. [20] of decreased uptake of Taxol in the presence of Cisplatin by making $\delta_{t}(C, T)$ a decreasing function of Cisplatin as described in Equation (7). Figure 7 shows the effect that increasing the parameter $\theta_{t}$ has on the additivity. Note that as $\theta_{r}$, increases, Cisplatin has more of an effect on decreasing the uptake of Taxol. We observe that the greater the effect of Cisplatin on Taxol uptake, the more antagonistic the combination. Again we observe that Taxol preceding Cisplatin is the more favorable sequence. We obtain very similar results when using the model parameters for the cell-line MCF-7/LY2.

Now we consider the pharmacodynamic effect described by Jones et al. [10] - Taxol increases

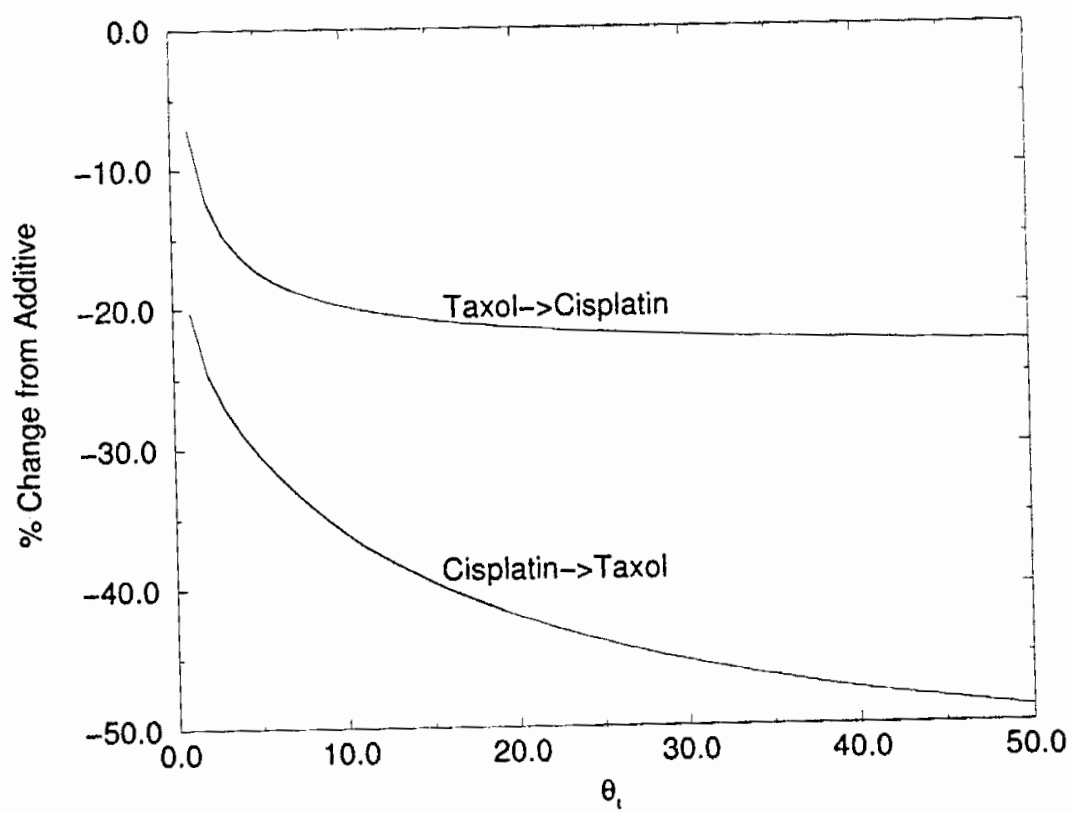

FIGURE 7 A2780 Parameters. Increased $\theta_{t}$ Represents Decreased Uptake of Taxol. 
the apoptotic rate. For our model increasing $\theta_{m}$ in Equation (8) increases the effect Taxol has on apoptosis. As can be seen in Figure 8, this effect gives us the first evidence for synergy in the model along with the fact that giving Taxol first remains the better combination according to the model. Figure 9 shows what happens as the time between doses (for Taxol before Cisplatin) is changed. For the A2780 cell line parameters this indicates that a delay of about a half a day between doses is the best due to the largest percent change from additivity. Figure 9 again shows the antagonistic effects of the sequence Cisplatin prior to Taxol. Finally, we observe the effects of altering dose with $\theta_{m}=50$. Figure 10 shows that smaller doses of Taxol followed by larger doses of Cisplatin have better synergic effects.

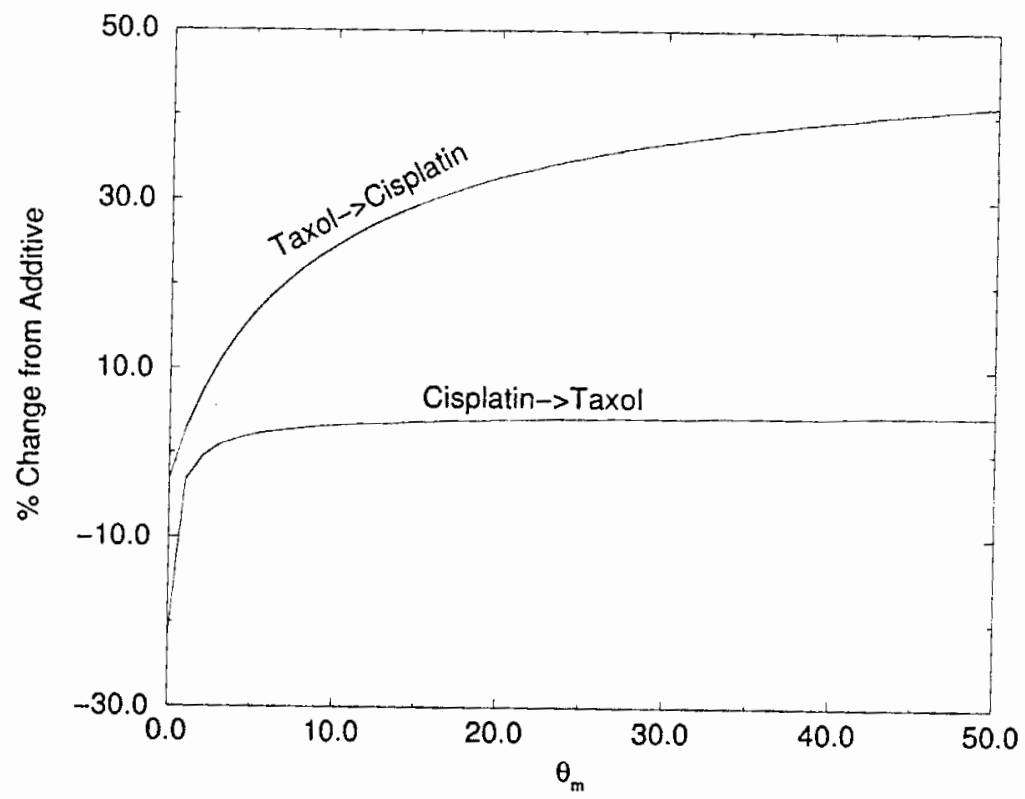

FIGURE 8 A2780 parameters. Increased $\theta_{m}$ represents increased apoptotic activity due to Taxol.
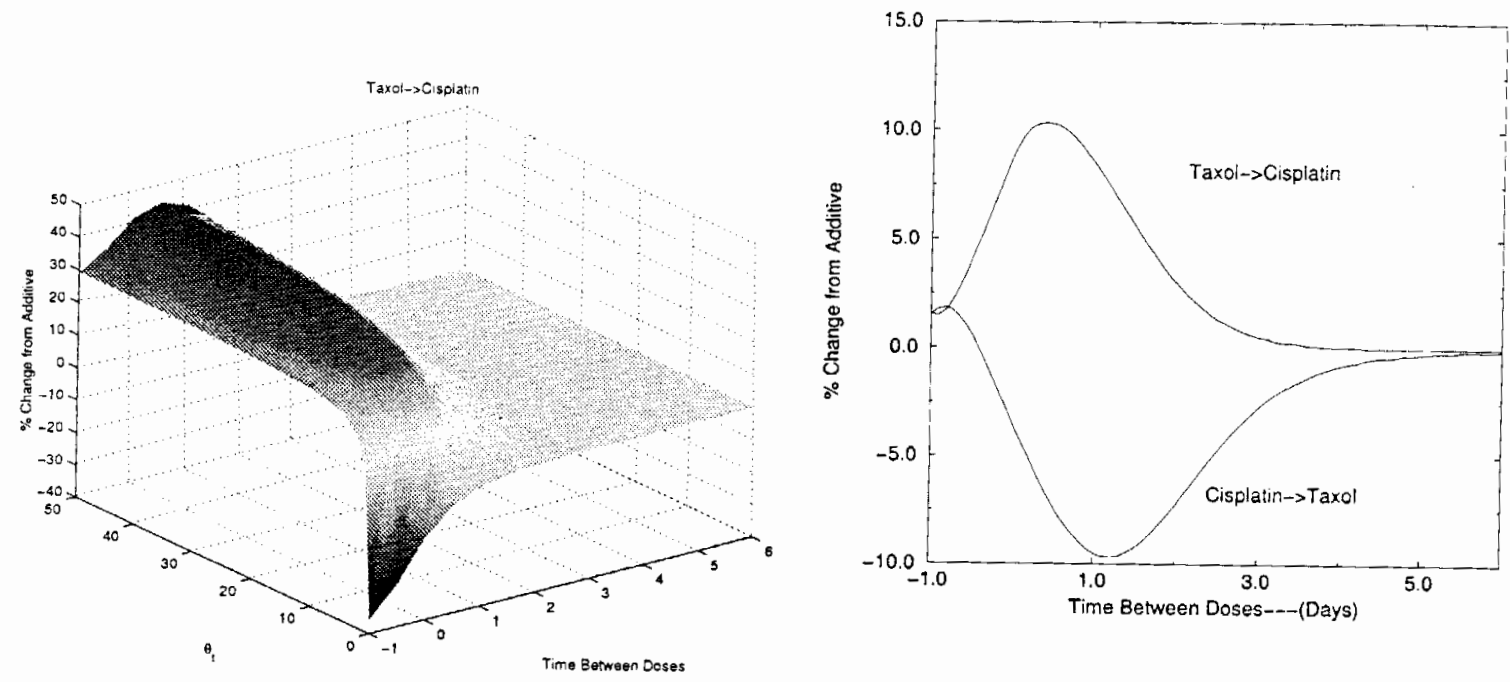

FIGURE 9 A2780 Parameters. 

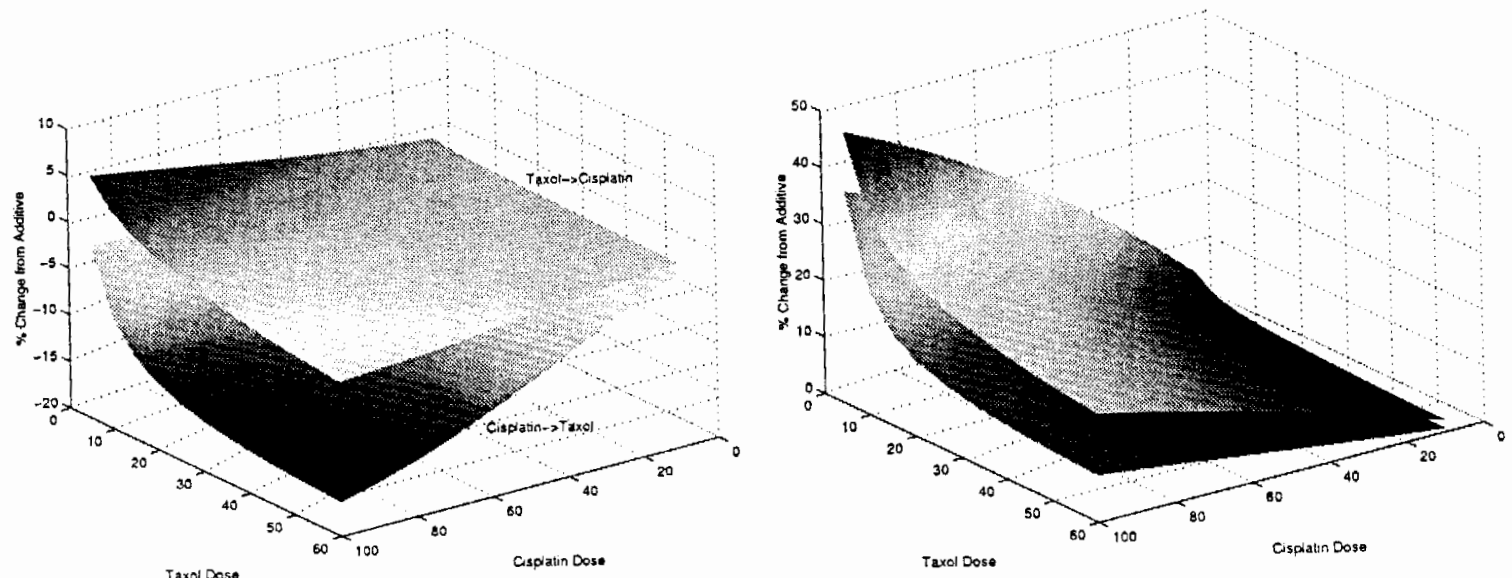

FIGURE 10 A2780 parameters. There is more DNA repair (i.e. more resistance to Cisplatin, larger $\eta$ ) in the right figure.

Again in all these cases it should be noted that the combination of Taxol given prior to Cisplatin is better.

\subsubsection{Pharmacokinetics}

Figure 11 shows the effects of increasing $\theta_{\lambda}$ in Equation 10 . Here we see that as we increase $\theta_{\lambda}$ (decrease the clearance of Taxol due to Cisplatin as described by Rowinsky et al. [17]) we see that the treatment for both Taxol prior to Cisplatin and Cisplatin prior to Taxol are both synergistic. But, as in all the previous cases, we observe that the model predicts that the administration of the Taxol before the Cisplatin to be the more effective regimen.

\section{DISCUSSION}

The combination of Cisplatin and Taxol is the current standard first-line chemotherapy in the treatment of advanced ovarian cancer. Clinical studies suggest that the optimal schedule for this combination is to administer the Taxol before the

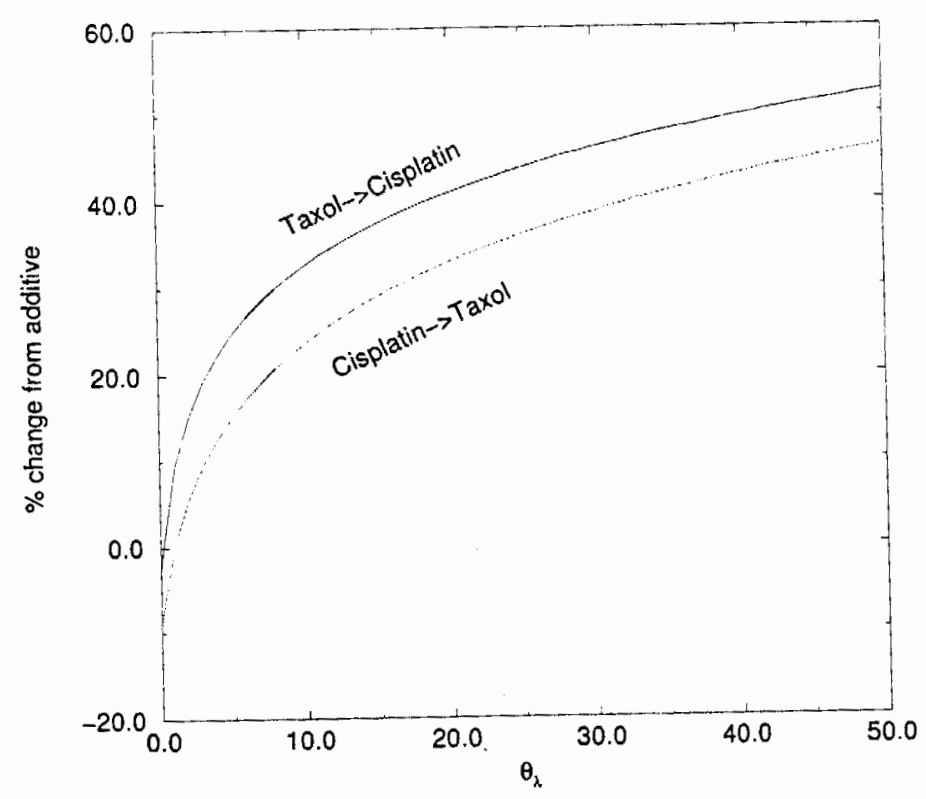

FIGURE 11 A2780 parameters. Increased $\theta_{\lambda}$ represents a decrease in Taxol clearance due to Cisplatin. 
Cisplatin. However, despite several clinical and laboratory studies, the reason for this scheduledependency remains unclear, and therefore one cannot be sure that this combination is being given optimally at present. Furthermore there are no data to confirm that the interaction is synergistic, or whether the schedule of Taxol before Cisplatin simply minimizes any antagonism between the agents.

Therefore, we have constructed a mathematical model in order to try and identify the mechanisms of any schedule-dependent interaction between Cisplatin and Taxol. The model therefore incorporates terms that reflect the likely cell-cycle dependent effects of these two drugs, as well as the currently identified pharmacokinetic and pharmacodynamic interactions. However, Figure 3 confirms that even in the absence of any pharmacological interactions, there is evidence of a schedule-dependent difference in efficacy, that appears to be consequent upon the particular phases of the cell-cycle in which the cells are active. Furthermore, adding the PD/PK effects does not alter the schedule dependence. This suggests that the schedule of Taxol before Cisplatin is fundamental to obtaining optimum efficacy, and the nature of any pharmacological or biological interactions may modulate this rather than be the primary cause.

Figure 7 demonstrates that any inhibition of Taxol uptake into tumor cells by prior administration of Cisplatin (as reported by Vanhoefer et al. [20]) results in significant antagonism. However it is interesting to note that the alternative schedule is still antagonistic but to a lesser degree. The data that suggest optimum efficacy when the drugs are administered around 24 hours apart (as seen in Figures 4 and 9) will considerably reduce this interaction. This is clearly something that could be done in the laboratory and the clinic, where both efficacy and intra-tumoural pharmacology could be investigated.

A more interesting suggestion perhaps, in terms of the mechanism of cell-killing (as reported by Jones et al. [10]), is the possibility that Taxol can induce bax expression and thus increase the likelihood of a cell undergoing apoptosis. Figure 8 shows quite clearly that this is predicted to result in synergy between the two drugs, as might be anticipated. This could be clinically relevant - there are conflicting data on the response and outcome for ovarian cancers that express mutated or abnormal p53, which normally serves to trigger apoptosis in a cell with damaged DNA. However the available data suggest that the outcome for patients whose tumors contain abnormal p53 is worse with Cisplatin therapy (Buttitta et al. [3]), but not necessarily worse when treated with Taxol (Smith-Srensen et al. [18]). If Taxol can not only induce apoptosis in a p53 independent manner (Debernardis et al. [6] and Menendez et al. [13]), but also increase the likelihood of a Cisplatin-treated cell to undergo apoptosis, then it may be possible to overcome some of the consequences of a mutant p53 and hence produce, at the very least, additive effects in combination with Cisplatin.

In determining the relevance and value of the model we must consider how sensitive the model results are to changes in the parameters. In order to investigate this, we have performed extra simulations of the model using different values for some of the parameters. Firstly, we considered changes in dose $(D)$ (cf. Figures 5 and 10). In these cases, we observed that the changes we made did not alter the conclusions. Next, we considered changes in $\alpha$ and $\beta$ (cf. Figure 6). It was observed that these changes had minimal effect on the AGD and again did not alter the conclusions. Finally, we made changes to the parameters $\delta_{t}, \gamma_{m}$, and $\lambda_{t}$ via changes in $\theta_{t}, \theta_{m}$, and $\theta_{\lambda}$ respectively (cf. Figures $7-9$, and 11 ). As in all the previous cases, these changes did not alter the model conclusion that Taxol prior to Cisplatin is the more effective sequence. Though this is by no means an exhaustive search of the complete parameter space, it does indicate that changes in some of the more important model parameters resulted in no changes to the conclusions.

Clinically one cannot be sure that administering the Taxol prior to Cisplatin results in synergy. However, knowledge of the most synergistic schedule of drug administration would potentially 
allow the best therapeutic strategy, and so use of this model can help predict possible schedules that appear more or less synergistic than others. It is however interesting to note that the model predicts an improved therapeutic effect for Taxol being administered before Cisplatin, and suggests an interval of one day between drug administrations to be optimal.

Clinical confirmation of these data will of course be difficult to achieve. However, studying various ovarian cancer cell lines in vitro gives one the possibility of determining whether or not the proposed schedules do produce the predicted synergy and/or antagonism. Such studies are currently in progress and will seek to determine in vitro the accuracy of the predicted drug schedule effects of the mathematical model. They may also help identify the importance of pharmacodynamic and cell-cycle interactions, but by necessity cannot consider any pharmacokinetic effects. However, such studies may help eliminate insignificant interactions so that a simpler model can be developed which can then be tested prospectively in patients.

In conclusion, this mathematical model of the combination of Taxol and Cisplatin appears to give results consistent with the current limited clinical and experimental data, and suggests that true synergy is only scen when Taxol increases the sensitivity of cells to Cisplatin by favorable changes in the balance of the intracellular proteins that regulate apoptosis. This would suggest that further improvement in the therapeutic efficacy may be achieved by strategies that alter the control of cellular responses to cytotoxics rather than more complicated clinical drug scheduling, although the truth of this observation awaits testing in both model systems and the clinic.

\section{References}

[1] Susan, G. Arbuck, Andrew Dorr and Michael, A. Friedman. (1994). Paclitaxel (taxol) in breast cancer. Breast Cancer, 8(1), 121-140.

[2] Berenbaum, M. C. (1989). What is synergy? Pharmacol. Rev., 41, 93-142.
[3] Buttitta, F., Marchetti, A., Gadducci, A., Pellegrini, S., Morganti, M., Carnicelli, V., Cosio, S., Gagetti, O., Genazzani, A. R. and Bevilacqua, G. (1997). p53 alterations are predictive of chemoresistance and aggressiveness in ovarian carcinomas: a molecular and immunohistochemical study. Br. J. Cancer, 75, 230-235.

14] Chou, T. C. and Talalay, P. (1986). Quantitative analysis of dose-effect relationships: the combined effects of multiple drugs or enzyme inhibitors. Adv. Enzyme Reg., 22, 27-55.

[5] de Vos, A. I., Nooter, K., Verwejj, J., Loos, W. J., Brouwer, E., deBruijn, P., Ruijgrok, E. J., van der Burg, $M$. E. L., Stoter, G. and Sparreboom, A. (1997). Differential modulation of cisplatin accumulation in leukocytes and tumor cell lines by the paclitaxel vehicle Cremophor EL. Annals of Oncology, 8, 1145-1150.

[6] Debernardis, D., Sire, E. G., De Feudis, P., Vikhanskaya, F., Valenti, M., Russo, P., Parodi, S., D'Incalci, M. and Broggini, M. (1997). p53 status does not affect sensitivity of human ovarian cancer cell lines to paclitaxel. Cancer Res., 57, 870-874.

[7] Engblom, P., Rantanen, V., Kulmala, J. and Grenman, S. (1996). Paclitaxel and cisplatin sensitivity of ovarian carcinoma cell lines tested with the 96-well plate clonogenic assay. Anticancer Research, 16(4A), 1743-1747.

[8] Greco, R. W., Bravo, G, and Parsons, J. C. (1995). The search for synergy: A critical review from a response surface perspective. Pharmacological Reviews, 47(2), $33 \mathfrak{l}-38.5$.

[9] Jekunen, A. P., Christen, R. D., Shalinsky, D. R. and Howell, S. B. (1994). Synergistic interaction between cisplatin and taxol in human ovarian carcinoma cells. in vitro. British Journal of Cancer, 69(2), 299-306.

[10] Jones, N. A., Turner, A. J., Mcllwrath, J., Brown, R and Dive, C. (1998). Cisplatin- and paclitaxel-induced apoptosis of ovarian carcinoma cells and the relationship between bax and bak up-regulation and the functional status of p53. Molecular Pharmacology, 53(5), 819-826.

[11] Levasseur, L. M., Greco, W. R., Rustum, Y. M. and Slocum, H. K. (1997). Combined action of paclitaxel and cisplatin against wildtype and resistant human ovarian carcinoma cells. Cancer Chemother. Parmacol., 40, 495-505.

[12] Liebmann, J., anf Fisher, J. E., Teague, D. and Cook, J. A. (1994). Sequence dependence of paclitaxel (taxol) combined with cisplatin or alkylators in human cancer cells. Oncology Research, 6(1), 25-31.

[13] Menendez, A. T., Laidlaw, J., Raventos-Suarez, C., Gelbert, L., Granas, A., Li, M. and Kramer, R. (1997). Taxol induces apoptosis by a $553 / \mathrm{p} 21$ independent mechanism in cisplatin resistant ovarian carcinoma cells (meeting abstract). Proc. Annu. Meet. Am. Assoc. Cancer Res., 38, A628.

[14] Milross, C. G., Peters, L. J., Hunter, N. R., Mason, K. A and Milas, L. (1995). Sequence-dependent antitumor activity of paclitaxel (taxol) and cisplatin in vivo. International Journal of Cancer, 62(5), 599-604.

[15] John Carl Panetta. (1997). A mathematical model of breast and ovarian cancer treated with paclitaxel. Math. Biosci., 146(2), 89-113.

[16] Parker, R. J., Dabholkar, M. D., Lee, K. B., BostickBruton, F. and Reed, E. (1993). Taxol effect on cisplatin sensitivity and cisplatin cellular accumulation in human ovarian cancer cells. Journal of the National Cancer Institute, $15,83-88$.

[17] Rowinsky, E. K., Gilbert, M. R., McGuire, W. P., Noe, D. A., Grochow, L. B., Forastiere, A. A., Ettinger, D. S., 
Lubeiko, B. G., Clark. B., Sartorius, S. E., Cornblath, D. R. Hendricks, C. B. and Donehower, R. C. (1991). Sequences of taxol and cisplatin: a phase $\mathrm{i}$ and pharmacologic study. J. Clin. Oncol., 9(9), 1692-1703.

[18] Smith-Srensen, B., Kaern, J., Holm, R., Drum, A., Trope, C. and Brresen-Dale, A. L. (1998). Therapy effect of either paclitaxel or cyclophosphamide combination treatment in patients with epithelial ovarian cancer and relation to tp53 gene status. Br. J. Cancer, 78, 375-381.
[19] Caroline, M. Spencer. and Diana Faulds. (1994). Paclitaxel a review of its pharmacodynamic and pharmacokinetics properties and therapeutic potential in the treatment of cancer. Drugs, 48(5), 794-847.

[20] Vanhoefer, U., Harstrick, A., Wilke, H., Schleucher, N., Walles, H., Schroder, J. and Seeber, S. (1995). Scheduledependent antagonism of paclitaxel and cisplatin in human gastric and ovarian carcinoma cell lines. in vitro. European Journal of Cancer, 31 A(1), 92-97. 


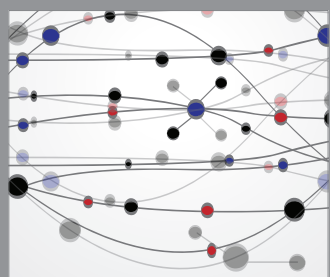

The Scientific World Journal
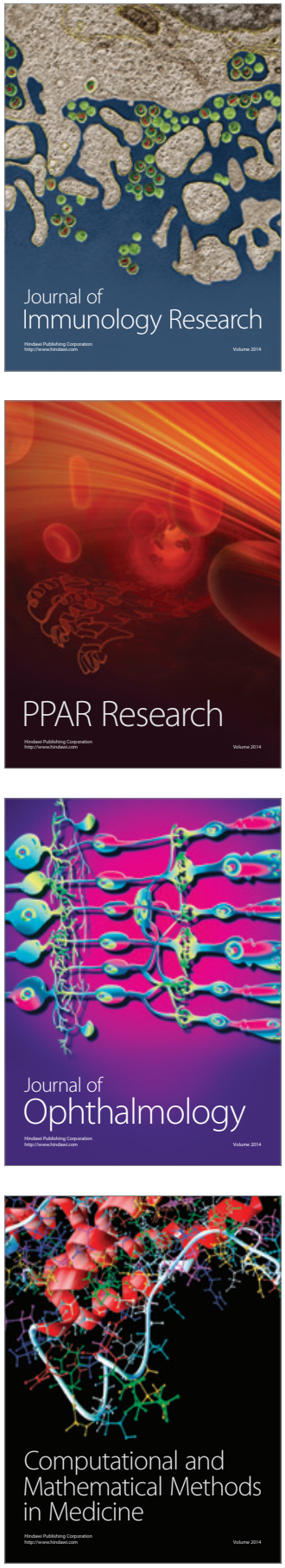

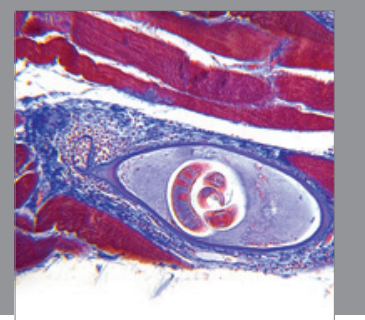

Gastroenterology

Research and Practice
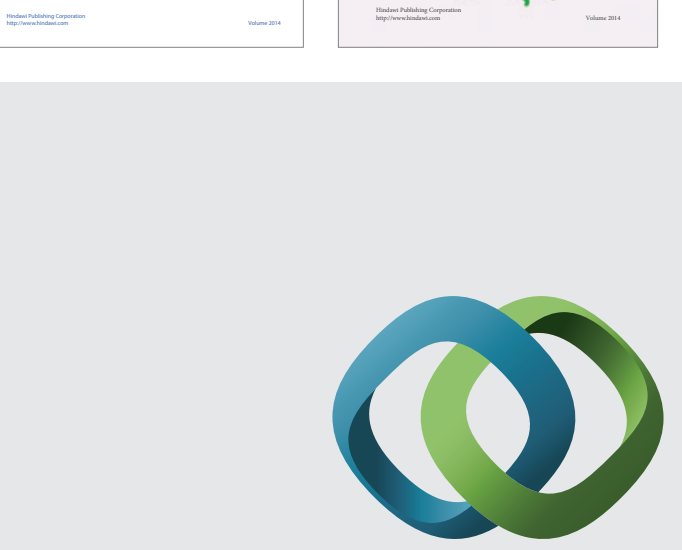

\section{Hindawi}

Submit your manuscripts at

http://www.hindawi.com
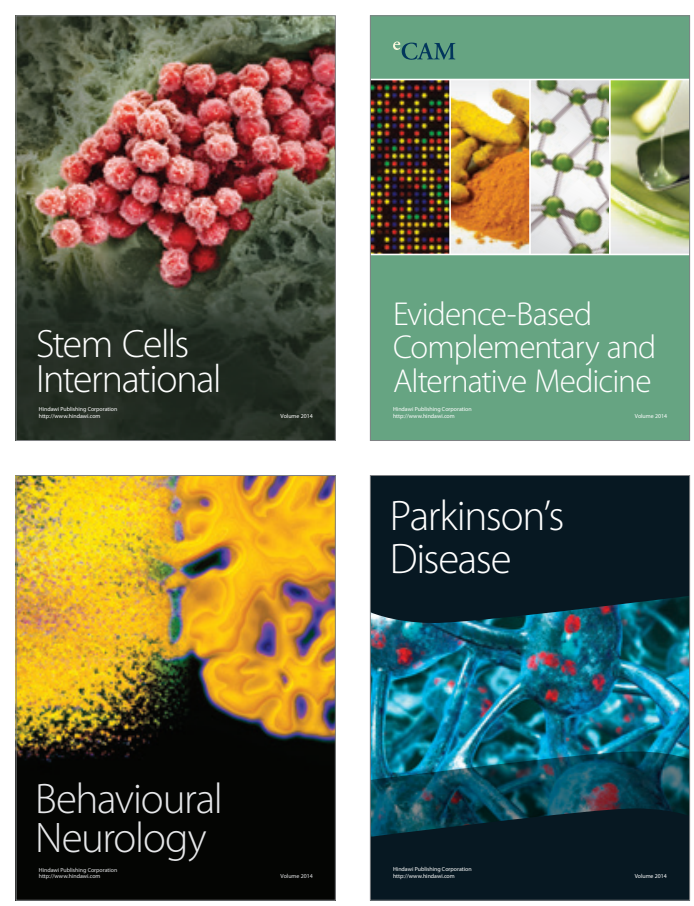

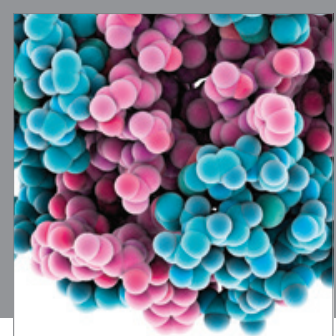

Journal of
Diabetes Research

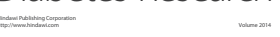

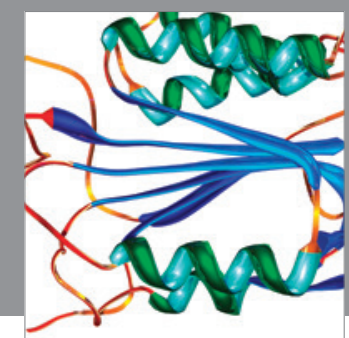

Disease Markers
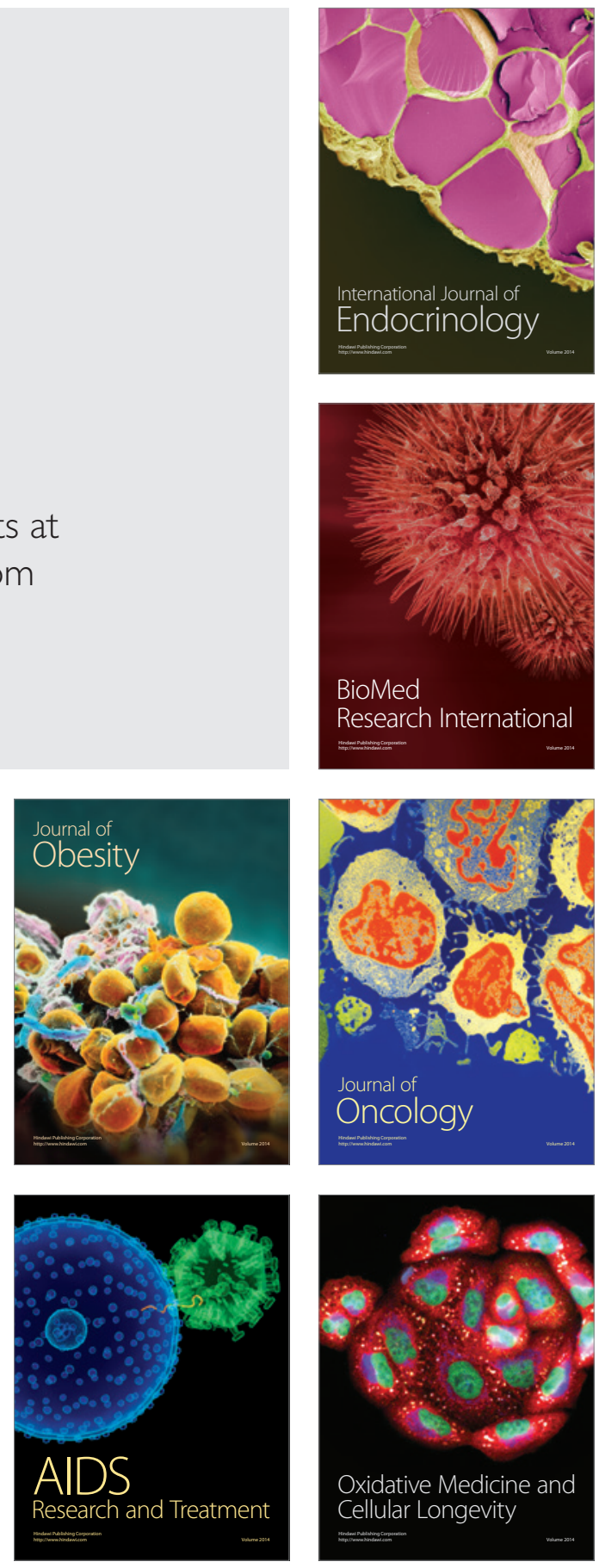